\title{
EIGENVALUE DISTRIBUTION OF TOEPLITZ MATRICES
}

\author{
PAUL G. NEVAI ${ }^{1}$
}

\begin{abstract}
A theorem of U. Grenander and G. Szegö on Toeplitz matrices is generalized. A new method is proposed for investigating eigenvalue distribution of Toeplitz matrices.
\end{abstract}

1. Let $d \alpha$ be a positive measure on the real line. Suppose that all the moments of $d \alpha$ are finite and the support of $d \alpha$ is an infinite set. Then there exist a sequence of polynomials $p_{n}(d \alpha, x)=\gamma_{n} x^{n}+\ldots, n=0,1,2, \ldots, \gamma_{n}>0$, such that

$$
\int_{-\infty}^{\infty} p_{k}(d \alpha, x) p_{l}(d \alpha, x) d \alpha(x)=\delta_{k l}
$$

If $\boldsymbol{g}$ is a real valued $d \alpha$-measurable function and all the moments of $g d \alpha$ are finite then we can form a matrix $T(g, d \alpha)=\left(a_{k l}\right)_{k, l=0}^{\infty}$ defined by

$$
a_{k l}=\int_{-\infty}^{\infty} p_{k}(d \alpha, x) p_{l}(d \alpha, x) g(x) d \alpha(x) .
$$

Such a matrix $T(g, d \alpha)$ is called Toeplitz matrix corresponding to $d \alpha$ and generated by $g$. For $n=1,2, \ldots$ the truncated matrix $T_{n}(g, d \alpha)$ is defined by $T_{n}(g, d \alpha)=$ $\left(a_{k l}\right)_{k, l=0}^{n-1}$. Since $T_{n}(g, d \alpha)$ is Hermitian, its eigenvalues $\Lambda_{k n}(g, d \alpha), k=1,2, \ldots, n$, are all real.

The purpose of this paper is to investigate the behavior of $\Lambda_{k n}$ as $n \rightarrow \infty$. This problem is a very old one and it has many applications in mathematics and physics. Let us just mention the book [3] of U. Grenander and G. Szegö that is devoted to such questions. In particular, Theorem 7.7(b) of [3] states that if $d \alpha$ is in the Szegö class, that is $\operatorname{supp}(d \alpha)=[-1,1]$ and $\log \alpha^{\prime}(\cos \theta) \in L^{1}$, and $g$ is continuous in $[-1,1]$ then

$$
\lim _{n \rightarrow \infty} \frac{1}{n} \sum_{k=1}^{n} G\left(\Lambda_{k n}(g, d \alpha)\right)=\frac{1}{\pi} \int_{-1}^{1} \frac{G(g(x))}{\left(1-x^{2}\right)^{1 / 2}} d x
$$

whenever $G$ is continuous. A correct proof of a somewhat more general form of this theorem was given in [4, p. 55]. In this paper I will show that the condition on $g$ can be relaxed and the same conclusion holds if $g$ only belongs to $L^{\infty}$. Note that $C$ is not dense in $L^{\infty}$ so that continuity arguments cannot be used in the process of generalization. More precisely, I will have to introduce a new method of investigating distribution of eigenvalues of Toeplitz matrices. The following result is basically

Received by the editors September 3, 1979.

AMS (MOS) subject classifications (1970). Primary 42C99, $15 A 18$.

Key words and phrases. Toeplitz matrix, eigenvalue, orthogonal polynomials.

'This material is based upon work supported by the National Science Foundation under Grant No. MCS 78-01868. 
a combination of the condition of Theorem 5.2(b) and the conclusion of Theorem 7.7(b) of [3].

Theorem 1. Let $\operatorname{supp}(d \alpha)=[-1,1]$ and $\alpha^{\prime}(x)>0$ for almost every $x \in[-1,1]$. Assume that the Toeplitz matrix $T(g, d \alpha)$ is generated by an $L^{\infty}$ function $g$. Let $G$ be a continuous function in an interval containing the essential range of $g$. Then the eigenvalues $\Lambda_{k n}$ of the truncated matrix $T_{n}(g, d \alpha)$ satisfy

$$
\lim _{n \rightarrow \infty} \frac{1}{n} \sum_{k=1} G\left(\Lambda_{k n}\right)=\frac{1}{\pi} \int_{-1}^{1} \frac{G(g(x))}{\left(1-x^{2}\right)^{1 / 2}} d x .
$$

Note that the proof of this theorem does not require the use of Erdös-Turan's celebrated result $[1$, p. 547] on the distribution of zeros of orthogonal polynomials. Actually, the case $g(x)=x$ of Theorem 1 is equivalent to the theorem of Erdös and Turan. I think that the proof of Theorem 1 is more elementary than any known proof of the Erdös-Turan theorem. Theorem 1 is an easy consequence of the following result which is likely to have other applications as well. The function $K_{n}(d \alpha, x, t)$ below is defined by

$$
K_{n}(d \alpha, x, t)=\sum_{k=0}^{n-1} p_{k}(d \alpha, x) p_{k}(d \alpha, t) .
$$

THEOREM 2. Let $d \alpha$ be such that $\operatorname{supp}(d \alpha)=[-1,1]$ and $\alpha^{\prime}(x)>0$ almost everywhere in $[-1,1]$. Suppose that $f$ belongs to $L^{\infty}$ in the square $[-1,1] \times[1,1]$ and satisfies

$$
\lim _{\varepsilon \rightarrow 0} \frac{1}{\varepsilon} \int_{x}^{x+\varepsilon}|f(x, t)-f(x, x)| d t=0
$$

for almost every $x \in(-1,1)$. Then

$$
\lim _{n \rightarrow \infty} \frac{1}{n} \int_{-1}^{1} \int_{-1}^{1} K_{n}^{2}(d \alpha, x, t) f(x, t) d \alpha(x) d \alpha(t)=\frac{1}{\pi} \int_{-1}^{1} \frac{f(x, x)}{\left(1-x^{2}\right)^{1 / 2}} d x
$$

I do not know of any result which would help to find out whether a given Toeplitz matrix $T(g, d \alpha)$ is generated by a continuous function $g$. On the other hand, Theorem 7.7(b) of [3] assumes continuity of $g$. The following result justifies Theorem 1 since it decides whether $T(g, d \alpha)$ is generated by an $L^{\infty}$ function $g$.

TheOReM 3. Let $T(g, d \alpha)$ be a Toeplitz matrix corresponding to da supported in $[-1,1]$ and satisfying $\alpha^{\prime}>0$ almost everywhere in $[-1,1]$. Let $m$ and $M$ be defined by

$$
m=\inf _{n, k}\left\{\Lambda_{k n}(g, d \alpha)\right\}, \quad M=\sup _{n, k}\left\{\Lambda_{k n}(g, d \alpha)\right\}
$$

Then the essential range of $g$ lies between $m$ and $M$. In particular, if both $m$ and $M$ are finite then $g$ is an $L^{\infty}$ function. Furthermore, if

$$
\sup _{l} \sum_{k=0}^{\infty}\left|\int_{-1}^{1} p_{k}(d \alpha, x) p_{l}(d \alpha, x) g(x) d \alpha(x)\right|<\infty
$$

then $g \in L^{\infty}$. 
It might be interesting to note that the converse statement

$$
\text { ess inf } g<m<M<\text { ess sup } g
$$

is well known and trivial.

2. This section is devoted to the proof of Theorems 1-3. Besides (1) the following notation will be used:

$$
\lambda_{n}(d \alpha, x)=K_{n}^{-1}(d \alpha, x, x) .
$$

Proof of Theorem 2. Let $d v$ denote the Chebyshev measure of $[-1,1]$, that is, let $\operatorname{supp}(d v)=[-1,1]$ and $v(x)=\int_{-1}^{x} d t /\left(1-t^{2}\right)^{1 / 2}$ for $x \in[-1,1]$. For $i=1,2$ define $g_{i}$ by

$$
g_{i}(x, t)=1+\|f\|_{\infty}+(-1)^{i} f(x, t) .
$$

Our first goal is to prove

$$
\lim _{n \rightarrow \infty} \lambda_{n}(d v, x) \int_{-1}^{1} K_{n}^{2}(d v, x, t) g_{i}(x, t)^{-1} d \alpha(t)=\left(1-x^{2}\right)^{1 / 2} \alpha^{\prime}(x) g_{i}(x, x)^{-1}
$$

for almost every $x \in[-1,1]$. Let $d \beta$ denote the sum of the singular and jump components of $d \alpha$. By Lemma 6.2.31 of [4, p. 92]

$$
\lim _{n \rightarrow \infty} \lambda_{n}(d v, x) \int_{-1}^{1} K_{n}^{2}(d v, x, t) d \beta(t)=0
$$

almost everywhere in $[-1,1]$. Since by (3) $0<g_{i}^{-1}<1$, this implies

$$
\lim _{n \rightarrow \infty} \lambda_{n}(d v, x) \int_{-1}^{1} K_{n}^{2}(d v, x, t) g_{i}(x, t)^{-1} d \beta(t)=0
$$

for almost every $x \in[-1,1]$. Thus to prove (4) we need to show

$$
\lim _{n \rightarrow \infty} \lambda_{n}(d v, x) \int_{-1}^{1} K_{n}^{2}(d v, x, t) g_{i}(x, t)^{-1} \alpha^{\prime}(t) d t=\left(1-x^{2}\right)^{1 / 2} \alpha^{\prime}(x) g_{i}(x, x)^{-1}
$$

almost everywhere in $[-1,1]$. Let $h_{i}$ be defined by

$$
h_{i}(x, t)=g_{i}(x, t)^{-1} \alpha^{\prime}(t)\left(1-t^{2}\right)^{1 / 2} .
$$

Since $\lambda_{n}(d v, x) \int_{-1}^{1} K_{n}^{2}(d v, x, t) d t /\left(1-t^{2}\right)^{1 / 2}=1$, formula (5) is equivalent to

$$
\lim _{n \rightarrow \infty} \lambda_{n}(d v, x) \int_{-1}^{1} K_{n}^{2}(d v, x, t)\left[h_{i}(x, t)-h_{i}(x, x)\right] d t /\left(1-t^{2}\right)^{1 / 2}=0 .
$$

Note that the integrability of $\alpha^{\prime}$ and formulas (2), (3) and (6) imply

$$
\lim _{\varepsilon \rightarrow 0} \frac{1}{\varepsilon} \int_{x}^{x+\varepsilon}\left|h_{i}(x, t)-h_{i}(x, x)\right| d t=0
$$

for almost every $x \in(-1,1)$. Now fix $x$ for which (8) holds and choose $\delta>0$ so that $-1<x-\delta<x+\delta<1$. Using the well-known estimate $\lambda_{n}(d v, x) K_{n}^{2}(d v, x, t)<3 \min \left\{n,(n(x-t))^{-2}\right\}[2$, pp. 106-108] we obtain 


$$
\begin{aligned}
\mid \lambda_{n}(d v, x) & \int_{-1}^{1} K_{n}^{2}(d v, x, t)\left[h_{i}(x, t)-h_{i}(x, x)\right] \frac{d t}{\left(1-t^{2}\right)^{1 / 2}} \mid \\
< & 3 n \int_{x-1 / n}^{x+1 / n}\left|h_{i}(x, t)-h_{i}(x, x)\right| \frac{d t}{\left(1-t^{2}\right)^{1 / 2}} \\
& +\frac{3}{n} \int_{1 / n<|x-t|<\delta} \frac{\left|h_{i}(x, t)-h_{i}(x, x)\right|}{(x-t)^{2}} \frac{d t}{\left(1-t^{2}\right)^{1 / 2}} \\
& +\frac{3}{n \delta^{2}} \int_{|x-t|>\delta ;-1<t<1}\left[\left|h_{i}(x, t)\right|+\left|h_{i}(x, x)\right|\right] \frac{d t}{\left(1-t^{2}\right)^{1 / 2}} \equiv I_{1}+I_{2}+I_{3}
\end{aligned}
$$

provided that $1 / n<\delta$. Applying (8) we get $\lim I_{1}=0$ as $n \rightarrow \infty$. Formula (6) implies that $I_{3}$ also tends to 0 as $n \rightarrow \infty$. $I_{2}$ can be estimated by using Lebesgue's method of integration by parts. Denoting by $C$ the maximum of $\left(1-t^{2}\right)^{-1 / 2}$ on $[x-\delta, x+\delta]$ we obtain

$$
\begin{aligned}
I_{3}<3 C\{n & \int_{x-1 / n}^{x+1 / n}\left|h_{i}(x, u)-h_{i}(x, x)\right| d u \\
& +\frac{1}{n \delta^{2}} \int_{x-\delta}^{x+\delta}\left|h_{i}(x, u)-h_{i}(x, x)\right| d u \\
& \left.+\frac{1}{n} \int_{1 / n<|x-t|<\delta}|x-t|^{-3}\left|\int_{x}^{t}\right| h_{i}(x, u)-h_{i}(x, x)|d u| d t\right\} .
\end{aligned}
$$

Thus by (8)

$$
\limsup _{n \rightarrow \infty} I_{3}<6 C \sup _{|\varepsilon|<\delta}\left|\frac{1}{\varepsilon} \int_{x}^{x+\varepsilon}\right| h_{i}(x, u)-h_{i}(x, x)|d u| .
$$

Therefore we get the inequality

$$
\begin{aligned}
\limsup _{n \rightarrow \infty} \mid \lambda_{n}(d v, x) & \int_{-1}^{1} K_{n}^{2}(d v, x, t)\left[h_{i}(x, t)-h_{i}(x, x)\right] \frac{d t}{\left(1-t^{2}\right)^{1 / 2}} \mid \\
& <6 C \sup _{|\ell|<\delta}\left|\frac{1}{\varepsilon} \int_{x}^{x+\varepsilon}\right| h_{i}(x, u)-h_{i}(x, x)|d u| .
\end{aligned}
$$

Now letting $\delta \rightarrow 0$ and using (8) we obtain (7). Having established (4) the theorem can easily be proved. Since $\alpha^{\prime}>0$ almost everywhere in $[-1,1]$ and $\lim _{n \rightarrow \infty}(1 / n) K_{n}(d v, x, x)=1 / \pi$ for $-1<x<1$ [4, p. 79], formula (4) implies

$$
\lim _{n \rightarrow \infty} \frac{1}{n} \frac{K_{n}^{2}(d v, x, x) \alpha^{\prime}(x)}{\int_{-1}^{1} K_{n}^{2}(d v, x, t) g_{i}(x, t)^{-1} d \alpha(t)}=\frac{1}{\pi} \frac{g_{i}(x, x)}{\left(1-x^{2}\right)^{1 / 2}}
$$

for almost every $x \in(-1,1)$. Thus by Fatou's lemma

$$
\begin{aligned}
\liminf _{n \rightarrow \infty} & \frac{1}{n} \int_{-1}^{1} \frac{K_{n}^{2}(d v, x, x) \alpha^{\prime}(x)}{\int_{-1}^{1} K_{n}^{2}(d v, x, t) g_{i}(x, t)^{-1} d \alpha(t)} d x \\
& >\frac{1}{\pi} \int_{-1}^{1} g_{i}(x, x) \frac{d x}{\left(1-x^{2}\right)^{1 / 2}} .
\end{aligned}
$$


The next step is to use the identity $K_{n}(d v, x, x)=\int_{-1}^{1} K_{n}(d v, x, t) K_{n}(d \alpha, x, t) d \alpha(t)$ and Schwarz's inequality to obtain

$$
K_{n}^{2}(d v, x, x)<\int_{-1}^{1} K_{n}^{2}(d v, x, t) g_{i}^{-1}(x, t) d \alpha(t) \int_{-1}^{1} K_{n}^{2}(d \alpha, x, t) g_{i}(x, t) d \alpha(t) .
$$

This inequality allows us to conclude that

$$
\begin{aligned}
\int_{-1}^{1} \frac{K_{n}^{2}(d \alpha, x, x) \alpha^{\prime}(x)}{\int_{-1}^{1} K_{n}^{2}(d v, x, t) g_{i}^{-1}(x, t) d \alpha(t)} d x \\
\quad<\int_{-1}^{1} \int_{-1}^{1} K_{n}^{2}(d \alpha, x, t) g_{i}(x, t) d \alpha(x) d \alpha(t)
\end{aligned}
$$

which together with (9) leads to

$$
\begin{gathered}
\liminf _{n \rightarrow \infty} \frac{1}{n} \int_{-1} \int_{-1} K_{n}^{2}(d \alpha, x, t) g_{i}(x, t) d \alpha(x) d \alpha(t) \\
>\frac{1}{\pi} \int_{-1}^{1} g_{i}(x, x) \frac{d x}{\left(1-x^{2}\right)^{1 / 2}} .
\end{gathered}
$$

Now applying the definition of $g_{i}(3)$ and the identities

$$
\frac{1}{\pi} \int_{-1}^{1} \frac{d x}{\left(1-x^{2}\right)^{1 / 2}}=\frac{1}{n} \int_{-1}^{1} \int_{-1}^{1} K_{n}^{2}(d \alpha, x, t) d \alpha(x) d \alpha(t)=1
$$

for $n=1,2, \ldots$, we get

$$
\begin{gathered}
\liminf _{n \rightarrow \infty}(-1)^{i} \frac{1}{n} \int_{-1}^{1} \int_{-1}^{1} K_{n}^{2}(d \alpha, x, t) f(x, t) d \alpha(x) d \alpha(t) \\
>(-1)^{i} \frac{1}{\pi} \int_{-1}^{1} \frac{f(x, x)}{\left(1-x^{2}\right)^{1 / 2}} d x
\end{gathered}
$$

for $i=1,2$. The last inequality is obviously equivalent to the statement of the theorem.

LeMMA 4. Let $T_{n}(g, d \alpha)$ be the truncated matrix of an arbitrary Toeplitz matrix $T(g, d \alpha)$. Then there exist $n$ polynomials $\varphi_{k n}(g, d \alpha, x)$ of degree at most $n-1$ such that

$$
\begin{gathered}
\int_{-\infty}^{\infty} \varphi_{k n}(g, d \alpha, x) \varphi_{l n}(g, d \alpha, x) d \alpha(x)=\delta_{k l} \\
\int_{-\infty}^{\infty} \varphi_{k n}(g, d \alpha, x) \varphi_{l n}(g, d \alpha, x) g(x) d \alpha(x)=\Lambda_{k n}(g, d \alpha) \delta_{k l}
\end{gathered}
$$

and

$$
\sum_{k=1}^{n} \varphi_{k n}(g, d \alpha, x) \varphi_{k n}(g, d \alpha, t)=K_{n}(d \alpha, x, t)
$$

Proof. Let $b_{k}=\left(b_{k}^{0}, b_{k}^{1}, \ldots, b_{k}^{n-1}\right), k=1,2, \ldots, n$, be a collection of orthonormalized eigenvectors of $T_{n}(g, d \alpha)$, that is $T_{n} b_{k}=\Lambda_{k n} b_{k}$ and $\left(b_{k}, b_{l}\right)=\delta_{k l}$. Define $\varphi_{k n}(x)$ by $\varphi_{k n}(x)=\sum_{j=0}^{n-1} b_{k}^{j} p_{j}(d \alpha, x)$. Then

$$
\int_{-\infty}^{\infty} \varphi_{k n}(x) \varphi_{l n}(x) d \alpha(x)=\left(b_{k}, b_{l}\right)=\delta_{k l}
$$


and

$$
\int_{-\infty}^{\infty} \varphi_{k n}(x) \varphi_{l n}(x) g(x) d \alpha(x)=\left(T_{n} b_{k}, b_{l}\right)=\Lambda_{k n} \delta_{k l} .
$$

Since the number of the polynomials $\varphi_{k n}$ is exactly $n$, the orthogonality relation (11) guarantees that $\sum_{k=1}^{n} \varphi_{k n}(x) \varphi_{k n}(t)$ is a reproduction kernel for the space of polynomials of degree at most $n-1$ supplied with the inner product of $L^{2}(d \alpha)$. Now (10) follows from the uniqueness of the reproduction kernel.

Proof of Theorem 1. Note that $f(x, t)=G(g(t))$ obviously satisfies (2) of Theorem 2. Thus, since

$$
\int_{-1}^{1} G(g(x)) K_{n}(d \alpha, x, x) d \alpha(x)=\int_{-1}^{1} \int_{-1}^{1} G(g(t)) K_{n}^{2}(d \alpha, x, t) d \alpha(x) d \alpha(t),
$$

the theorem will be proved if it can show the validity of

$$
\lim _{n \rightarrow \infty}\left\{\frac{1}{n} \sum_{k=1}^{n} G\left(\Lambda_{k n}\right)-\frac{1}{n} \int_{-1}^{1} G(g(x)) K_{n}(d \alpha, x, x) d \alpha(x)\right\}=0 .
$$

By Lemma 4 we have

$$
\begin{aligned}
\frac{1}{n} \sum_{k=1}^{n} G\left(\Lambda_{k n}\right) & -\frac{1}{n} \int_{-1}^{1} G(g(x)) K_{n}(d \alpha, x, x) d \alpha(x) \\
= & \frac{1}{n} \sum_{k=1}^{n} \int_{-1}^{1}\left[G\left(\Lambda_{k n}\right)-G(g(x))\right] \varphi_{k n}^{2}(x) d \alpha(x) \equiv I
\end{aligned}
$$

where $\varphi_{k n}(x)=\varphi_{k n}(g, d \alpha, x)$. Now fix $\varepsilon>0$ and choose $\delta>0$ so that $\mid G(x)-$ $G(y) \mid<\varepsilon$ for $|x-y|<\delta$. Then we can write

$$
\begin{aligned}
I= & \frac{1}{n} \sum_{k=1}^{n} \int_{\left|\Lambda_{k n}-g(x)\right|<\delta}\left[G\left(\Lambda_{k n}\right)-G(g(x))\right] \varphi_{k n}^{2}(x) d \alpha(x) \\
& +\frac{1}{n} \sum_{k=1}^{n} \int_{\left|\Lambda_{k n}-g(x)\right|>\delta}\left[G\left(\Lambda_{k n}\right)-G(g(x))\right] \varphi_{k n}^{2}(x) d \alpha(x) \equiv I^{1}+I^{2} .
\end{aligned}
$$

By the choice of $\delta$

$$
\left|I^{1}\right|<\varepsilon \frac{1}{n} \sum_{k=1}^{n} \int_{-1}^{1} \varphi_{k n}^{2}(x) d \alpha(x)=\varepsilon
$$

We also have

$$
\begin{aligned}
\left|I^{2}\right| & <2 \delta^{-2} \max |G| \frac{1}{n} \sum_{k=1}^{n} \int_{-1}^{1}\left(g(x)-\Lambda_{k n}\right)^{2} \varphi_{k n}^{2}(x) d \alpha(x) \\
& =2 \delta^{-2} \max |G| \frac{1}{n} \sum_{k=1}^{n} \int_{-1}^{1}\left(g^{2}(x)-2 g(x) \Lambda_{k n}+\Lambda_{k n}^{2}\right) \varphi_{k n}^{2}(x) d \alpha(x) .
\end{aligned}
$$

Using Lemma 4 we obtain

$$
\left|I^{2}\right|<2 \delta^{-2} \max |G| \frac{1}{n}\left\{\int_{-1}^{1} g^{2}(x) K_{n}(d \alpha, x, x) d \alpha(x)-\sum_{k=1}^{n} \Lambda_{k n}^{2}\right\} .
$$

Since $K_{n}(d \alpha, x, x)=\int_{-1}^{1} K_{n}^{2}(d \alpha, x, t) d \alpha(t)$ and

$$
\sum_{k=1}^{n} \Lambda_{k n}^{2}=\operatorname{Trace}\left(T_{n}^{2}(g, d \alpha)\right)=\int_{-1}^{1} \int_{-1}^{1} g(x) g(t) K_{n}^{2}(d \alpha, x, t) d \alpha(x) d \alpha(t),
$$


we can rewrite (14) in the form

$$
\left|I^{2}\right|<2 \delta^{-2} \max |G| \frac{1}{n} \int_{-1}^{1} \int_{-1}^{1}\left[g^{2}(x)-g(x) g(t)\right] K_{n}^{2}(d \alpha, x, t) d \alpha(x) d \alpha(t) .
$$

Note that the function $f(x, t)=g^{2}(x)-g(x) g(t)$ satisfies condition (2) of Theorem 2 and $f(x, x)=0$ almost everywhere in $[-1,1]$. Therefore $\lim _{n \rightarrow \infty} I^{2}=0$ holds. Taking (13) into account we get lim $\sup _{n \rightarrow \infty}|I|<\varepsilon$. Since $\varepsilon$ is arbitrary, $I$ tends to 0 as $n \rightarrow \infty$; that is (12) has been proved.

Proof of TheORem 3. It is well known that for every polynomial $\pi$

$$
\frac{\int_{-1}^{1} \pi^{2}(t) g(t) d \alpha(t)}{\int_{-1}^{1} \pi^{2}(t) d \alpha(t)}<M
$$

For $-1<x<1$ and $n=1,2, \ldots$, let $\pi(t)$ be defined by $\pi(t)=K_{n}(d v, x, t)$ where $d v$ denotes the Chebyshev measure of $[-1,1]$. Then by (15) the inequality

$$
\frac{\lambda_{n}(d v, x) \int_{-1}^{1} K_{n}^{2}(d v, x, t) g(t) d \alpha(t)}{\lambda_{n}(d v, x) \int_{-1}^{1} K_{n}^{2}(d v, x, t) d \alpha(t)}<M
$$

holds. It follows from Lemma 6.2 .32 of $[4$, p. 93] that the numerator and denominator in (16) converge to $\left(1-x^{2}\right)^{1 / 2} g(x) \alpha^{\prime}(x)$ and $\left(1-x^{2}\right)^{1 / 2} \alpha^{\prime}(x)$ respectively for almost every $x \in[-1,1]$ as $n \rightarrow \infty$. Since $\alpha^{\prime}(x)>0$ almost everywhere in $[-1,1]$, we obtain $g(x) \leqslant M$. Applying the same reasoning to $T(-g, d \alpha)$ we obtain $g(x)>m$ almost everywhere in $[-1,1]$. The last part of the theorem follows from the first one and from Theorem 1.18(b) of [3].

\section{REFERENCES}

1. P. Erdös and P. Turan, On interpolation. III, Ann. of Math. (2) 41 (1940), 510-553.

2. G. Freud, Orthogonale polynome, Akadémiai Kiadó, Budapest, 1969.

3. U. Grenander and G. Szegö, Toeplitz forms and their applications, Univ. of California Press, Berkeley and Los Angeles, 1958.

4. P. Nevai, Orthogonal polynomials, Mem. Amer. Math. Soc. No. 213, Amer. Math. Soc., Providence, R. I., 1979.

Drpartment of Mathematics, Ohio State University, Columbus, Ohio 43220 